\title{
Electrophysiologic Validation of Diffusion Tensor Imaging Tractography during Deep Brain Stimulation Surgery
}

\author{
(D) V.A. Coenen, (D) C. Jenkner, (D)C.R. Honey, and (D)B. Mädler
}

\begin{abstract}
BACKGROUND AND PURPOSE: Diffusion tensor imaging fiber tractography-assisted planning of deep brain stimulation is an emerging technology. We investigated its accuracy by using electrophysiology under clinical conditions. We hypothesized that a level of concordance between electrophysiology and DTI fiber tractography can be reached, comparable with published modeling approaches for deep brain stimulation surgery.
\end{abstract}

MATERIALS AND METHODS: Eleven patients underwent subthalamic nucleus deep brain stimulation. DTI scans and high-resolution T-- and T2-weighted MR imaging was performed at 3T. Corticospinal tracts were traced. We studied electrode positions and current amplitudes that elicited corticospinal tract effects during the operation to determine relative corticospinal tract distance. Postoperatively, 3D deep brain stimulation electrode contact locations and stimulation patterns were applied for the same corticospinal tract distance estimation.

RESULTS: Intraoperative electrophysiologic $(n=40)$ clinical effects in 11 patients were detected. The mean intraoperative electrophysiologic corticospinal tract distance was $3.0 \pm 0.6 \mathrm{~mm}$; the mean image-derived corticospinal tract distance (DTI fiber tractography) was $3.0 \pm 1.3 \mathrm{~mm}$. The $95 \%$ limits of agreement were $\pm 2.4 \mathrm{~mm}$. Postoperative electrophysiology $(n=44)$ corticospinal tract activation effects were encountered in 9 patients; 39 were further evaluated. Mean electrophysiologic corticospinal tract distance was $3.7 \pm 0.7 \mathrm{~mm} ;$ for DTI fiber tractography, it was $3.2 \pm 1.9 \mathrm{~mm}$. The $95 \%$ limits of agreement were $\pm 2.5 \mathrm{~mm}$.

CONCLUSIONS: DTI fiber tractography depicted the medial corticospinal tract border with proved concordance. Although the overall range of measurements was relatively small and variance was high, we believe that further use of DTI fiber tractography to assist deep brain stimulation procedures is advisable if inherent limitations are respected. These results confirm our previously published electric field simulation studies.

ABBREVIATIONS: CST = corticospinal tract; DBS = deep brain stimulation; EPio = intraoperative electrophysiology; EPpo = postoperative electrophysiology; $\mathrm{FT}$ = fiber tractography; STN = subthalamic nucleus

D TI fiber tractography (FT) to assist deep brain stimulation (DBS) emerges as an interesting technology in different indications for the treatment of chronic medically refractory

Received August 24, 2015; accepted after revision January 22, 2016.

From the Department of Stereotactic and Functional Neurosurgery (V.A.C., B.M.) and the Clinical Trial Unit (C.J.), Freiburg University Medical Center, Freiburg, Germany; Surgical Center for Movement Disorders/Division of Neurosurgery (C.R.H.) and Department of Physics and Astronomy (B.M.), University of British Columbia, Vancouver, British Columbia, Canada; and Philips Healthcare (B.M.), Hamburg, Germany.

Paper previously presented in part at: Congress of the European Society of Stereotactic and Functional Neurosurgery, September 17-20, 2014; Maastricht, the Netherlands.

Please address correspondence to Volker A. Coenen, MD, Department of Stereotactic and Functional Neurosurgery, Albert-Ludwigs-University, Freiburg, Breisacher Str 64, D-79106 Freiburg (i.Br.), Germany; e-mail: volker.coenen@uniklinik-freiburg.de

-- Indicates open access to non-subscribers at www.ajnr.org http://dx.doi.org/10.3174/ajnr.A4753 disorders. ${ }^{1,2}$ Several groups are now aware of the clinical benefits that arise from the application of this direct targeting technology. The true anatomic structures that translate into adverse effects of stimulation are often not understood. Very likely, DBS modulates fibers that can be visualized with DTI FT. This noninvasive imaging technology might directly show the structures on which DBS exerts its effects and might prove to be a promising technology in direct and individualized targeting for DBS. DTI FT-assisted DBS has already led to a better understanding of the treatment of tremor, Parkinson disease, pain, and depression. ${ }^{1-9}$ In the latter, it has led to the description of a completely new target region (the superolateral branch of the medial forebrain bundle)..$^{6,9,10}$

Before the application of DBS, DTI FT had become part of the standard armamentarium for microneurosurgical resections of eloquently located brain lesions. ${ }^{11,12}$ However, despite a study that showed the superiority of DTI-based neurosurgery for clinical 
outcome during resection in eloquent regions, ${ }^{13}$ there have also been reports of the inferiority of DTI FT to depict the true extension of the corticospinal tract (CST) during brain tumor surgery. ${ }^{14}$ Hahn et $\mathrm{al}^{15}$ and Nimsky et $\mathrm{al}^{16-18}$ have extensively investigated the application error of DTI FT and found it to be roughly $5 \mathrm{~mm}$ in the cortical region. In our own previous report, ${ }^{5}$ we have tried to assess the application error of DWI-based depiction of the deep-seated CST during deep brain stimulation surgery under anatomically "undistorted" conditions.

Intuitively, DBS surgery warrants an even higher accuracy than neuronavigated microneurosurgical approaches, but nevertheless visually controlled, because among other factors, the effective positioning of an electrode or probe predominantly relies on a geometrically accurate depiction of the target region with an imaging technology. For the DWI technology, the accuracy was determined to be $3 \mathrm{~mm}$ in the $\mathrm{z}$-direction (vertical). ${ }^{5}$ On the basis of our own experience in DTI-assisted DBS, we concluded that it is possible to use DTI FT to visualize target structures for functional stereotactic and neurosurgical procedures. ${ }^{4,6,8,19}$ However, as of today, a clear determination of the validity of a DTI-based depiction of fiber tracts during stereotactic and functional procedures and its rigorous evaluation with sound electrophysiologic methods is lacking in the literature.

We present a study that tries to give more insight into these problems. Applying 2 methods, intraoperative electrophysiologic determination of the CST border (EPio) based on a current spread model ${ }^{20}$ and postoperative electrophysiologic evaluation (EPpo) based on readily implanted DBS electrode positions, a finite element model and a voltage-driven approach, ${ }^{19,21}$ we aimed to determine an electrophysiologic validation of the DTI FT-based depiction of the CST during subthalamic nucleus (STN) DBS surgery. Taking all possible methodologic inaccuracies into account, we hypothesized that a level of concordance of 2-3 mm between electrophysiology and DTI FT can be reached, which justifies further use of our previously published modeling approaches for DBS surgery. ${ }^{19}$

\section{MATERIALS AND METHODS \\ Ethics}

This study received approval from the University of British Columbia clinical research ethics board (reference No. H06-04023). Patients gave written informed consent for participation in this study. The study followed the tenets of the Declaration of Helsinki.

\section{Patient Cohort}

Eleven patients underwent bilateral STN DBS surgery for advanced Parkinson disease ( 9 men; mean age, $55 \pm 9.6$ years) according to standardized selection guidelines. All patients had a preoperative levodopa challenge test evaluated with Part III of the Unified Parkinson's Disease Rating Scale with an improvement of $>40 \%$.

\section{Imaging Studies}

Preoperative MR imaging was conducted 1-3 months before the operation on a whole-body 3T MR imaging scanner (Intera; Philips Healthcare, Best, the Netherlands), equipped with a high- performance dual-mode gradient coil (maximum amplitude, $80 \mathrm{mT} / \mathrm{m}$; maximum slew rate, $200 \mathrm{~T} / \mathrm{m} / \mathrm{s}$ ) by using a 6 -element phased array head coil.

The examination was preceded by a quick T1-weighted survey and a parallel imaging reference scan. Anatomic data were obtained with a 3D MPRAGE sequence (3D T1 turbo field echo): axial FOV $=212 \mathrm{~mm}$ and $132 \mathrm{~mm}$ coverage in the superoinferior direction with an isotropic acquisition voxel size of $1 \mathrm{~mm}^{3}, \mathrm{TE}=$ $6 \mathrm{~ms}$, TR $=10 \mathrm{~ms}$, turbo-factor $=169$, linear profile order, inversion preparation with an adiabatic hyperbolic secant pulse, $\mathrm{TI}=$ $950 \mathrm{~ms}$, shot interval $=3000 \mathrm{~ms}$, sensitivity-encoding $=1.7$. For visualization of the area of the subthalamic nucleus, we used a multisection T2-weighted fast spin-echo sequence with similar FOVs and voxel dimensions. The coverage in superoinferior directions was reduced to $116 \mathrm{~mm}$ to focus on the area of the midbrain and its nuclei. Further parameters were the following: turbo factor $=15, \mathrm{TE}=80 \mathrm{~ms}$, linear profile order, $\mathrm{TR}=3000 \mathrm{~ms}$, sensitivity encoding $=2.0$. The concept of reduced refocusing angles $\left(120^{\circ}\right)$ was used to reduce the specific absorption rate and therefore scan time. The examination was concluded with a DTI scan for subsequent application of fiber tracking. Scan parameters for DTI were as follows: single-shot spin-echo EPI with secondorder shim, TE $=60 \mathrm{~ms}, \mathrm{TR}=10,500 \mathrm{~ms}, b=800 \mathrm{~s} / \mathrm{mm}^{2}$, FOV $=$ $212 \mathrm{~mm}$, matrix $=106 \times 106$ leading to an in-plane voxel size of $2 \times 2 \mathrm{~mm}^{2}$. We acquired 70 sections with $2-\mathrm{mm}$ thickness and no intersection gap to cover the entire brain. Diffusion encoding was performed in 15 noncollinear directions on an icosahedral geodesic grid to sample all spheric directions isotropically, followed by the non-diffusion-weighted reference scan (B0 image). All scans were performed in an axial orientation.

\section{Fiber Tracking of the CST}

Fiber tracking of the CST was performed in a deterministic approach as described before. ${ }^{22}$ In brief, the fractional anisotropy level was kept at 0.2. Minimal fiber length was set to $45 \mathrm{~mm}$. Seed density was held at 5.0. Maximal directional change of fibers was chosen between $35^{\circ}$ and $50^{\circ}$. The complete precentral gyrus served as the seed region for the fiber tracking. Identification of the precentral gyrus was based on the criterion of Yousry et al. ${ }^{23}$

\section{STN DBS Surgery and Postoperative Programming}

The detailed implantation procedure has been previously described. ${ }^{6}$ In brief, stereotactic implantation of DBS electrodes was performed with a stereotactic frame (Universal Compact Head Frame; Integra Radionics, Burlington, Massachusetts) with the patient under local anesthesia. A combined micro-/macroelectrode (FHC MME; Medtronic, Minneapolis, Minnesota) was inserted into the brain by using a microTargeting Drive (Medtronic). Microelectrode recordings followed by macrostimulation studies were performed. All patients had DBS electrodes placed bilaterally and received an implantable impulse generator (Kinetra neurostimulator; Medtronic) under general anesthesia during the same procedure. Postoperatively, all patients underwent a helical 3D CT approximately 6 weeks after the operation to corroborate the final STN DBS electrode location. 


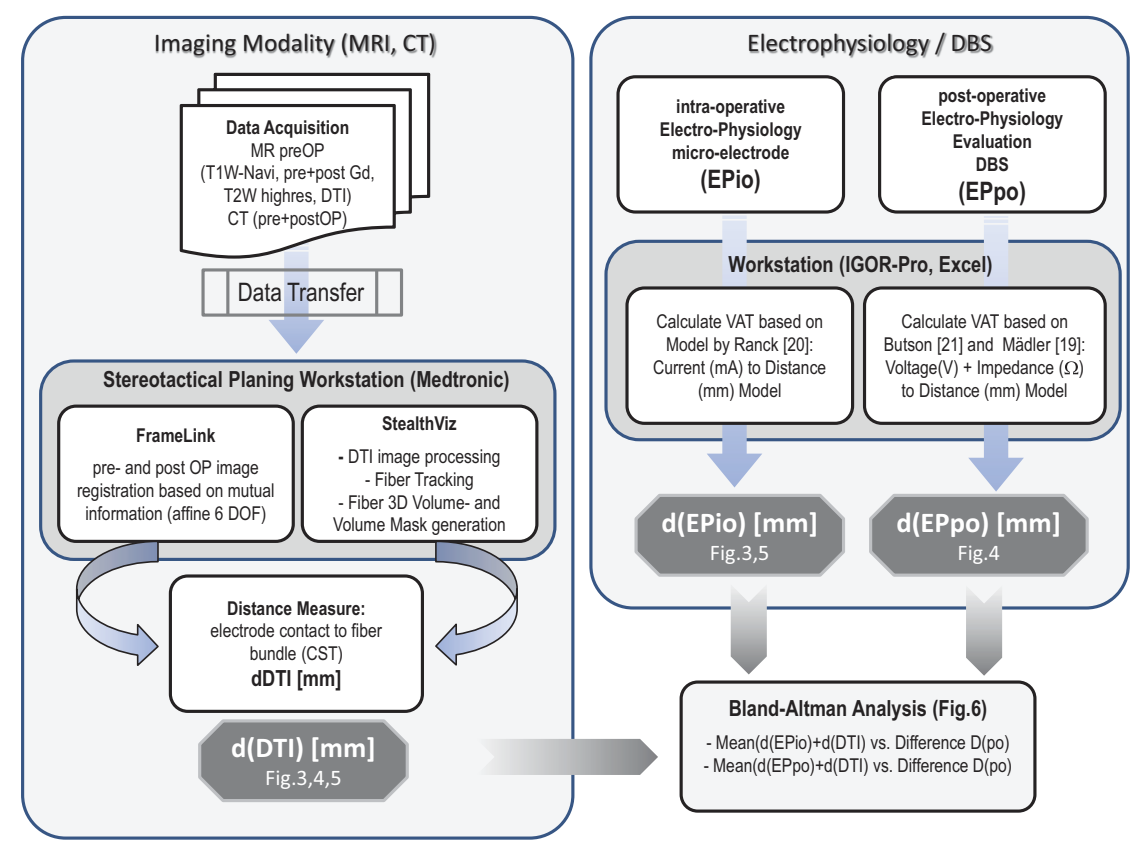

FIG 1. Flow chart of the procedures. Post OP indicates postoperative; preOP, preoperative; DOF, deformation; EPio, intraoperative electrophysiology; EPpo, postoperative electrophysiology; Navi, Navigation (Sequence); pre, before; post, after; VAT, volume of activated tissue; highres, high-resolution.
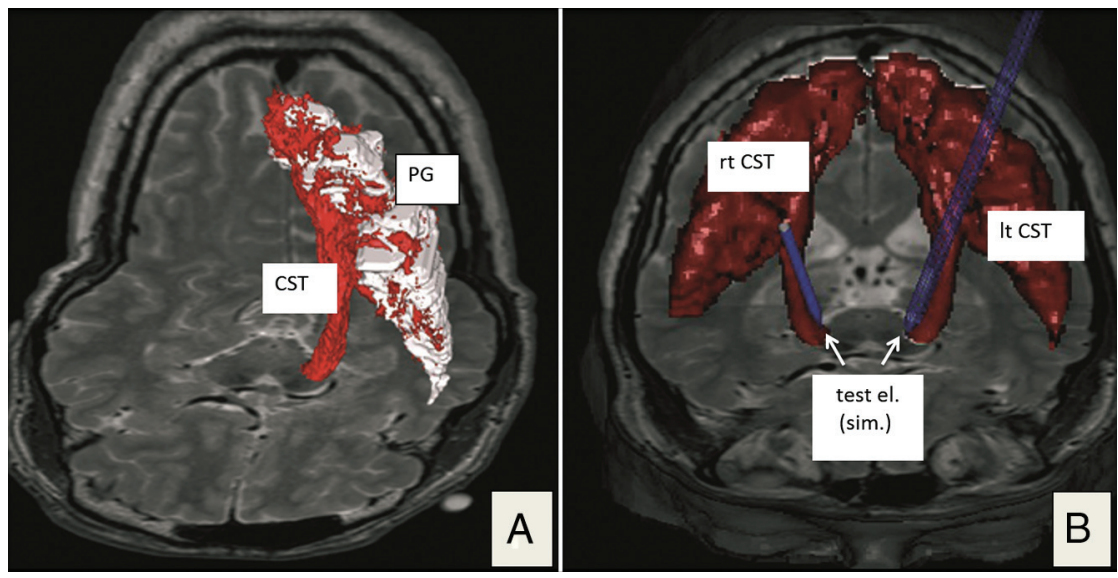

FIG 2. 3D renditions of the corticospinal tract. $A$, Depiction of a left CST (red) in the fiber-tracking software (StealthViz DTI; Medtronic) but already depicted as a DICOM hull structure. B, Bilateral visualization of the transferred DICOM structure in the planning software (FrameLink 5.0; Medtronic Surgical Navigation). Blue probe simulations indicate intraoperatively tested electrode positions (test el.). PG indicates precentral gyrus; test el. (sim.), simulated test electrode position. trophysiologic distance compared with the DTI FT detection of the CST, respectively. ${ }^{5}$ According to Ranck, ${ }^{20}$ a power law distance-to-current relationship of the volume of activated tissue could be shown for various myelinated fibers when stimulated with a monopolar electrode setting. From the empiric data (Fig 1 in $\operatorname{Ranck}^{20}$ ), we were able to derive a generalized power law relationship (linear relationship on double logarithmic scaling) between the applied current and the diameter of the volume of activated tissue for clinically relevant settings (applied current in the milliampere regimen) and hence a predictor of the dimensions for the relevant electromagnetic field used for stimulation. The minimal distance in millimeters at which stimulation settings cause neurologic adverse events for this intraoperative setting is called $\mathrm{d}$ (EPio) (Fig 1, right column). ${ }^{20}$

\section{Second Experiment: EPpo and Detection of the Medial Internal Capsule/CST Border Based on DBS Electrodes}

The preoperative 3T MR imaging studies and postoperative 3D CT data were integrated in the StealthViz DT software application (Medtronic Navigation, Louisville, Colorado) on a stand-alone Linux workstation (Intel, Santa Clara, California), by using the automatic fusion mode of the software. The fusion quality was inspected visually and was scrutinized appropriately for further analysis in every case. Evaluation of a relevant effective electrode contact with a capsular adverse event was performed from fused CT data (Fig 3) and was expressed relative to the midcommissural point coordinates (Fig 4). After identifying each effective electrode contact from CT, we determined its location

\section{First Experiment: EPio and Detection of the Medial CST Border}

Experimental settings are explained in Fig 1. After mapping the STN location, we performed macrostimulation to confirm a contralateral clinical benefit (reduction of rigidity, reduced bradykinesia) at a low threshold ( $\leq 1 \mathrm{~mA}, 100 \mu \mathrm{s}, 130 \mathrm{~Hz}$ ) and a high threshold for adverse events $(>3 \mathrm{~mA})$. Constant current stimulation was applied. The adverse events were typically of a capsular nature (indicating the medial border of the internal capsule). These effects were contralateral facial contractions, contraction of the arm and hand, capsular dysarthria, or conjugate eye-movement disorders. These effects and the respective electrode position were noted for later simulation and evaluation (Fig 2) of the elec- with respect to the STN (as determined by the high-resolution T2-weighted MR imaging) (Fig 3) and its shortest distance to the CST (as displayed with fiber tracking) (Fig 3A). Clinical effects and capsular adverse events were tested with increments of $0.5 \mathrm{~V}$ in a voltage-constant stimulation mode. Therapeutic impedances were measured during capsular responses (see above) to allow an estimation of electric field sizes ${ }^{19}$ and thus the electrophysiologic distance to the CST, according to the work of Butson et al. ${ }^{21}$

As elaborately described in Mädler et $\mathrm{al},{ }^{19}$ we adopted a simple model to estimate the volume of activated tissue based on a monopolar stimulation design of the DBS electrode, by fitting a $2 \mathrm{D}$ polynomial to the empiric data obtained by Butson 

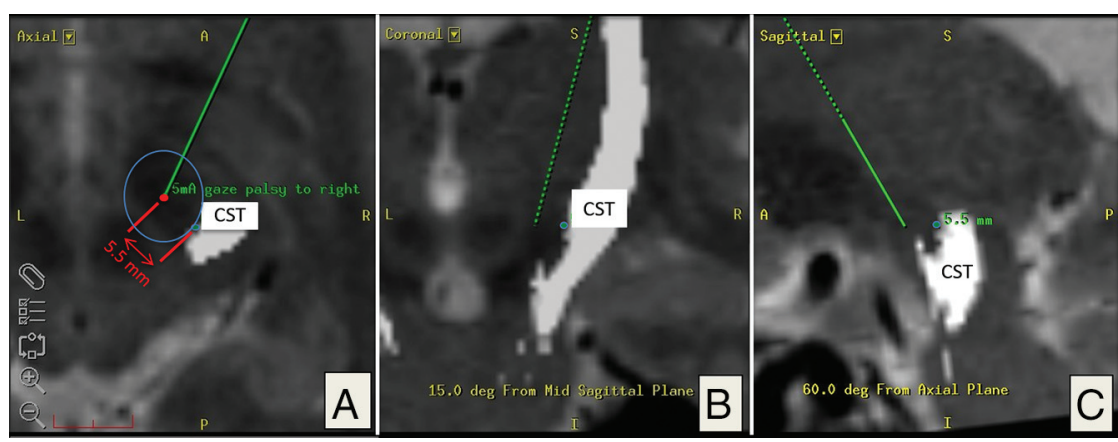

FIG 3. Evaluation based on intraoperative electrophysiology: corticospinal tract depiction in axial, coronal, and sagittal $(A-C)$ planes. $A$, Red dot indicates post hoc simulation of the intraoperative position of the test electrode in the planning software (Framelink 5.0; Medtronic Surgical Navigation) according to microTargeting Drive settings. In this example, $5 \mathrm{~mA}$ of intraoperative stimulation resulted in "gaze palsy" as capsular effect. The shortest spatial distance to the medial CST border of $5.5 \mathrm{~mm}$ is indicated with a blue circle. Both coordinates (electrode tip, medial border of CST) were recorded and later plotted (Fig 4). Note that the CST is located posterior and lateral relative to the positon of the electrode $(A)$.
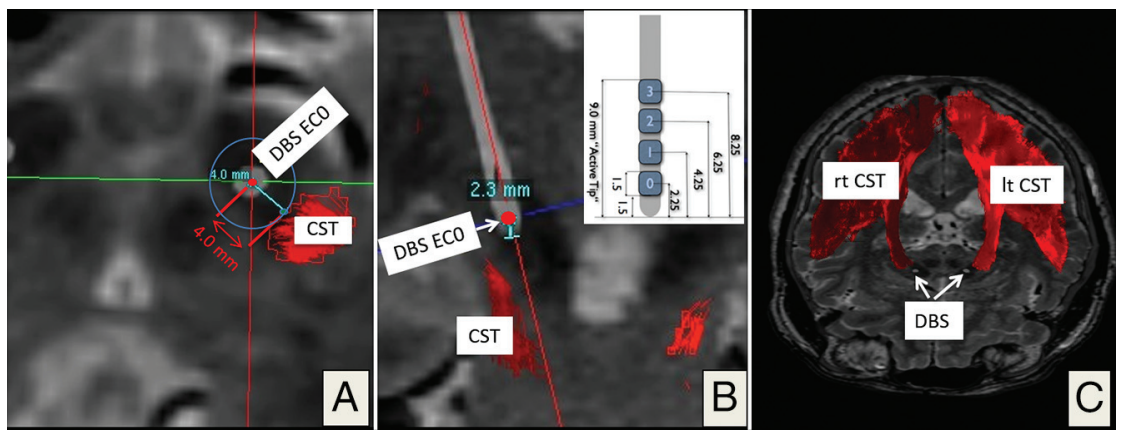

FIG 4. Postoperative electrophysiologic evaluation by using CT depiction of the DBS electrode artifacts. The 3D helical postoperative CT is superimposed on the planning data. Reconstruction along the main DBS electrode (white) axis, quasiaxial $(A)$ and coronal $(B)$. The minimal spatial distance to the medial border of the CST is $4.0 \mathrm{~mm}$. $B$, The DBS electrode (inset; geometry; DBS lead model 3389; Medtronic) is seen as a white structure in the STN region. In this example, electrode contact 0 ( $E C O$, deepest contact, $2.3 \mathrm{~mm}$ from the electrode tip) elicited capsular effects during postoperative clinical testing. C, 3D rendering of the right (rt) and left (lt) CSTs with DBS electrode artifacts from helical CT.

et al. ${ }^{21}$ Input parameters are the stimulation voltage at the DBS contact and the measured impedance for this electrode placement in the patient. The resulting parameter is the diameter of the volume of activated tissue, which can be geometrically placed around the actual electrode contact. $d(E P p o)$ is the radius of the volume of activated tissue in millimeters under stimulation settings (voltage and impedance) when the electromagnetic field touches the CST and causes the described neurologic adverse events (Fig 1).

\section{Statistics and Methods for Comparison of Experimental Results}

A Bland-Altman (or Tukey mean) difference plot is a common modus operandi to analyze the agreement between 2 different measurement methods, ${ }^{24}$ in our case distance measurements performed with DTI FT and intraoperative electrophysiology as well as DTI FT compared with postoperative electrophysiology. Because there are multiple measurements per patients, an adjustment proposed by Bland and Altman $(2007)^{25}$ was used for the calculation of the SD. A 95\% concordance level is the average difference $\pm 1.96^{\star} \mathrm{SD}$ of the difference and is a measure showing whether 2 separate measurement acquisitions are congruent. Correlation analysis by using Spearman correlation coefficients are applied to give further insight.

\section{RESULTS \\ Clinical}

The preoperative Parkinson medication was reduced postoperatively by $45 \%$ from baseline in combination with the initiation of stimulation, indicating successful STN DBS surgery. No worsening of the patients occurred in the immediate postoperative period. Clinically relevant effective electrode contacts were within the limits given in the literature for the sensorimotor STN.

\section{First Experiment (EPio)}

Intraoperatively, we elicited 40 CST adverse events in 11 patients (with multiple measurements per patient at different electrode positions): conjugate forced eye deviation ( $n=15)$; face contraction $(n=10)$; throat contraction, partly with dysarthria $(n=8)$; dysarthria $(n=4)$; unilateral forced eye opening $(n=1)$; foot contraction $(n=1)$; and hand contraction $(n=1)$ between 2 and $5 \mathrm{~mA}(3 \pm 1.3 \mathrm{~mA})$. In the post hoc analysis, these adverse events were analyzed on the basis of the simulated electrode position and the DTI-based rendition of the CST (Fig 2). The electrophysiologic distance to the internal capsule was $2.9 \pm 0.6 \mathrm{~mm}$, as calculated by Ranck $(1975)^{20}$; the mean image-derived distance (DTI FT) was $3.0 \pm 1.3 \mathrm{~mm}$. The mean average difference (bias) between the 2 measurements was $0.0 \pm 1.2$. This led to limits of agreement of $\pm 2.44 \mathrm{~mm}$. The Spearman correlation between the measurements was 0.34 (Fig $5)$.

\section{Second Experiment (EPpo)}

Forty-four internal capsule effects were encountered during postoperative programming (multiple measurements per patient): dysarthria $(n=19)$, facial contraction $(n=15)$, hand contraction $(n=3)$, forced eye deviation $(n=5)$, and throat contraction $(n=2)$. Therapeutic impedances were measured during initial programming. Due to limitations of the finiteelement electric field estimation model (limitations in voltage and impedances), ${ }^{19,21} 39$ adverse events in 9 patients could be further evaluated. The electrophysiologic distance to the CST was $3.7 \pm 0.6 \mathrm{~mm}$. The imaging-derived distance based on DTI FT between the effective electrode contact and the medial bor- 
axial

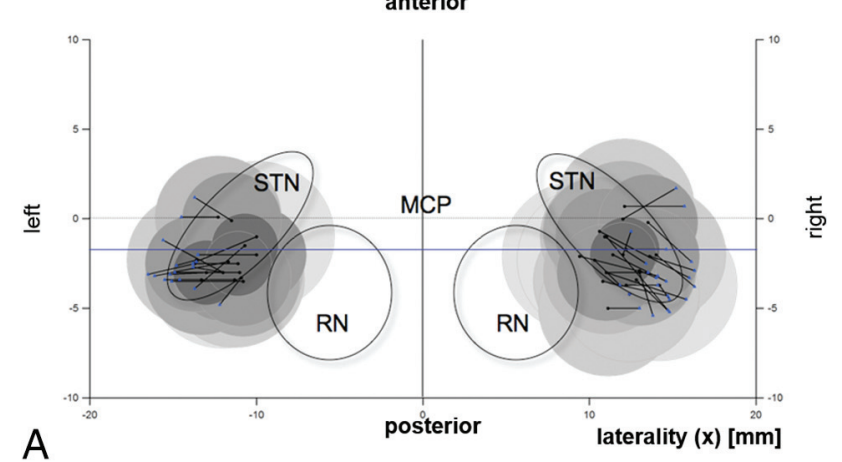

A

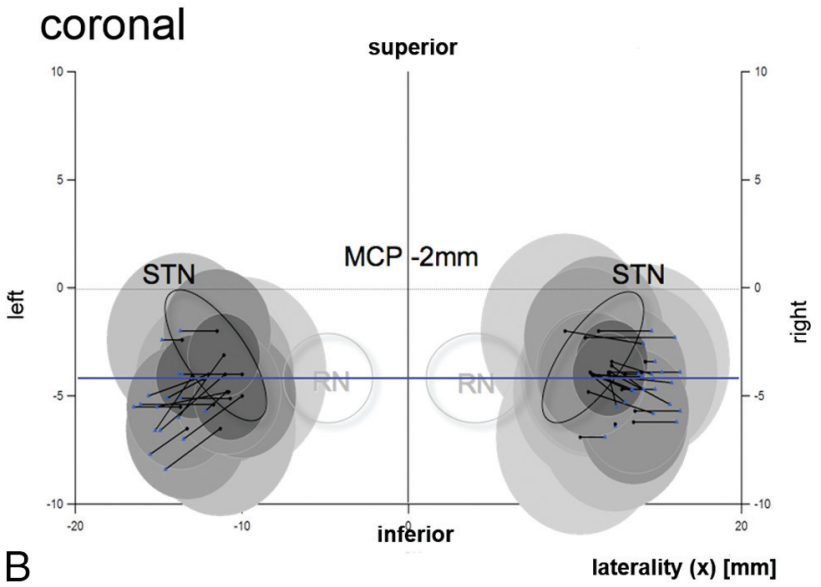

FIG 5. Graphic depiction of the EPio experiment results. Atlas templates in axial (A, $6.2 \mathrm{~mm}$ below the midcommissural point [MCP]) and coronal sections ( $B, 2 \mathrm{~mm}$ behind the MCP) (idealized according to Schaltenbrand and Wahren ${ }^{46}$ ). Intraoperative electrode positions are represented by black dots. Black lines represent the shortest distance in space to the CST as depicted with the DTI technology. Blue dots show the individual CST penetration in space. Shaded circles indicate estimated volumes of activated tissue around a test electrode, specific to the current that was applied to elicit an electrophysiologic CST response according to Ranck (1975), (Fig 1). ${ }^{20}$ CST (blue dots) corresponds nicely with electric field borders, indicating that medial CST definition with DTI reliably predicts the CST border as measured with electrophysiology. RN indicates red nucleus. (Of note in $A$, the CST is always located posterior and lateral to the STN region.)

der of the CST was $3.3 \pm 1.6 \mathrm{~mm}$. The mean average difference (bias) between the 2 measurements was $0.4 \pm 1.3$. This led to limits of agreement of $\pm 2.5 \mathrm{~mm}$. The Spearman correlation between the measurements was 0.44 .

\section{DISCUSSION}

The application of the DTI FT-based delineation of the CST in its most proximal, thus cortical, parts during microsurgical resection has long been debated. The localization error determined was 5 $\mathrm{mm}$ and led to the application of "sheath regions" surrounding fiber tracts at risk, which artificially maximized the extension of the cross-sectional diameter of the CST. ${ }^{17,18,26-28}$ With this approach, functional integrity could be spared in the vicinity of the CST. Examples in the literature showed that purely relying on fiber-tract delineation without safety margins led to a detrimental patient outcome. ${ }^{14}$ With a root square mean error of distortion of 3-5 mm for neuronavigation (depending on the use of CT or MR imaging, respectively), the accuracy demands for the DTI FT- based depiction of the CST and other fiber structures are obviously lower for neuronavigated interventions than for functional stereotactic procedures. The success of functional stereotactic procedures is, among other factors, mainly based on accurate imaging. The localization accuracy of stereotactic frames ranges between 1.5 and $2 \mathrm{~mm} .{ }^{29,30}$ However the vector error can be as high as $3.15 \mathrm{~mm}$. Thus, a new imaging technique should probably not be far outside this accuracy range. However, with the DTI FTassisted DBS approach, structures that were merely not known or simply not directly visible (dentatorubrothalamic tract, medial forebrain bundle) became readily targetable regions that otherwise would have to be explored and found by literally hunting through the brain on multiple paths with additional bleeding risks.

We have described the use of a comparable imaging technology, DWI, in a rather similar setting of STN DBS surgery. ${ }^{5}$ Other groups have tried to approach the accuracy level of DTI in clinical DBS procedures and have come to the conclusion that the use of DBS surgery with this technology cannot be advised. ${ }^{31}$ While this conclusion might be true, in our opinion, these authors based their results on imaging data, software use, and a study setup that was not geared to looking at the specific questions asked. ${ }^{32}$ Therefore, a specific approach designed to look at this problem, albeit still in a clinical setting, appeared appropriate.

Other groups used approaches rather similar to ours to look at the electrophysiologic effects of stimulation of the internal capsule. ${ }^{33,34}$ Duerden et $\mathrm{al}^{34}$ used an approach in which they retrospectively mapped capsular effects (muscle contractions of different body parts) to MR imaging anatomy (albeit not DTI). The aim of this study was to draw conclusions on the capsular topography in the posterior limb and to generate a probabilistic electrophysiologic data base. With their curved electrode used for lesioning, the authors could directly map fibers of the internal capsule with good spatial accuracy. Their electrophysiologic map represents the typical topographic representation of muscle groups (face toward the knee of the internal capsule, leg toward the posterior aspect). ${ }^{34}$ Chaturvedi et $\mathrm{al}^{33}$ published an interesting study, again with a different angle on capsular anatomy. In a single patient undergoing STN DBS, they used electromyographic recordings to show activations of distinct muscle groups. They used detailed simulation models based on the DTI-based tissue anisotropy and inhomogeneity. In their computation, cable models of axonal pathways were shaped. They debated the use of a simple voltagedistance approach. According to their data, it is likely that a DBS electrophysiologic model that does not take the interactions between (anisotropic) tissue and electric field into account will likely overestimate the actual current spread. ${ }^{34}$

In our study, the results of the Bland-Altman plots (Fig 6) give hints of a good concordance of the 2 measurements (DTI and electrophysiology). However, in our measurements, the range of distances was small and thus the relative error seems high. This is predominantly because there was no dramatic displacement of an electrode during measurements and naturally the CST can only be maximally $1-5 \mathrm{~mm}$ away from any stimulation point (the STN is a target region close to the CST). The Spearman correlations of 0.34 (EPio) and 0.44 (EPpo) only showed a trend toward correlation between electrophysiology and imaging. 

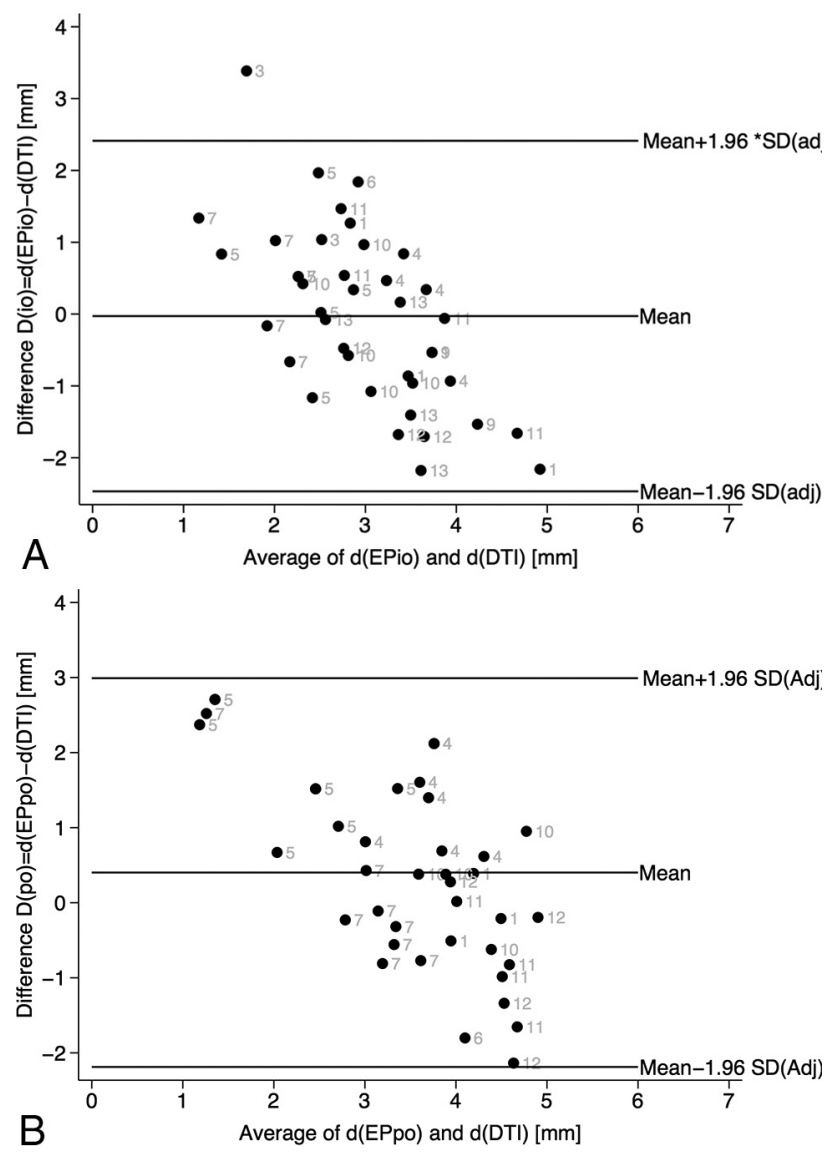

FIG 6. A, Bland-Altman plot, intraoperative measurements. Gray labels indicate individual patients. $B$, Bland-Altman plot, postoperative measurements. Gray labels indicate individual patients.

However, when cautiously interpreting our results, we can state the following: With the use our approach, a concordance of 2.44-2.5 mm, respectively, was found between DTI FT and electrophysiologic macrostimulation. It is important to carefully discuss these concordance levels in light of the methods applied and the interpretations they allow.

If one assumes that electrophysiology resembles a kind of criterion standard in depicting neuronal structures in the living human brain, a concordance level might allow an extrapolation toward the application accuracy of DTI FT. The concordance levels are not the same as the accuracy of the method but might help reflect on it.

The resolution of MR imaging itself is in the range of $2 \mathrm{~mm}$. However, in light of previous studies, which came to the conclusion that the neuronavigation localization error (root square mean error of distortion) of DTI is $5 \mathrm{~mm}$, our extrapolated localization error $(2.5 \mathrm{~mm})$ is much better and actually almost within the error of a frame-based stereotactic system itself (which can be up to $2 \mathrm{~mm}$; the vector error can be up to $3.15 \mathrm{~mm}$ ). Moreover, our own clinical experience with the DTI FT-assisted approach is such that the actual error ranges are lower than the $2.4-2.5 \mathrm{~mm}$ found here. However, our analysis would likely warrant a larger sample to allow a mere "shrinkage" of the limits of agreement to clearly prove our assumptions true. If these error ranges are taken together, we would cautiously suggest that the concordance levels detected here are acceptable in the context of DTI FT-assisted
DBS. The evaluation itself shows that the 2 methods (electrophysiology and DTI) are congruent for the distance measurements for which they were applied.

We and other groups have already applied this technology to directly target fiber tracts with improved clinical benefit for the patients, ${ }^{1-4,6,8,9,35,36}$ to scrutinize the adverse events of DBS $^{1,6,19,35}$ or to develop new target regions for DBS surgery. ${ }^{9,10}$ All these applications almost render the present study outdated. However, this study might help to establish solid ground for any of these efforts with respect to electrophysiologic validation of the DTI technology in the functional and stereotactic neurosurgical setting.

\section{Limitations}

Limitations of DTI-based fiber tracking are related to the design of the DTI sequence itself, its various methods of data acquisition, limited signal-to-noise ratio, partial volume effects, and the intrinsically large size of intraplanar voxels during in vivo DTI applications. Furthermore, there are limitations of the described fiber assignment by continuous tractography algorithm. There might be ambiguities in following the correct connection pathways in areas of crossing, kissing, or branching fibers. These ambiguities are clearly a limitation of the single diffusion tensor model, combined with a relatively low spatial resolution (approximately 2-mm isotropic voxel dimension). ${ }^{37-39}$ Nevertheless, these results from deterministic fiber tracking appear to be justified if anatomic descriptions of displayed fiber tracts such as the CST can be followed. However, the results of the DTI FT application can be rather diverse. ${ }^{22}$ This diversity, among other factors, depends on the software used.

Deterministic versus Probabilistic Algorithms. We were dealing with patients with Parkinson disease. This, in itself, limits the scanning time for more sophisticated approaches such as DTI for probabilistic approaches. To our knowledge, no software is validated (and certified) with probabilistic tracking in the context of surgical treatment.

The accuracy of fusion is very difficult to interpret. It is especially difficult to determine, for EPpo, the accuracy of the localization of the DBS electrode. Visually performed accuracy checks appear to show good agreement when landmarks in different imaging modalities (CSF spaces, vessels, bony landmarks) are used to compare the validity of the fusion. In the clinical context, this is everyday practice, for example, if preoperative nonstereotactic MR imaging is fused to a stereotactic CT scan. In this respect, this is a valid approach. The fusion accuracy of MR imaging and postoperative CT has previously been determined to be $0.5 \mathrm{~mm}$ for the localization of DBS electrodes from postoperative CT. ${ }^{40,41}$ This accuracy, however, is only part of the overall accuracy of this part of our evaluation method (EPpo). The fusion software applied here to fuse CT and MR imaging (T1, T2, DTI sequences) uses an affine algorithm with $6 d f$. To our knowledge, certified surgical planning systems are not yet equipped with algorithms that compensate for local deformations (typically for DTI) with elastic registration (eg, in the region of the anterior pons or in the frontal lobe, close to the corpus callosum). However, we do not think that these deformations play a major role for visualization of the CST or other fiber structures in the midbrain. We have used the post- 
operative determination of electrode positions with CT multiple times in previous work. ${ }^{1,4,6-8,10,42,43}$

Accuracy of Simulation of the Intraoperative Test Electrode Position. The accuracy of the determination of the position of the macrotip of the test electrode (EPio) will be dependent on the accuracy of the stereotactic frame $\mathrm{e}^{29,30}$ and thus will be $1.5-2 \mathrm{~mm}$. Due to air entering the CSF spaces, brain shift might occur during the operation, theoretically leading to a wrong positioning of the test or DBS electrodes. ${ }^{44}$ We and other groups have found that brain shift only plays a minor role in displacements of DBS electrodes and can be prevented by sealing the CSF space. ${ }^{45}$ Intraoperative lateral fluoroscopy, which is regularly performed during the operation, did not show any deviation of the microelectrodes during the operation. However, a second plane (anteroposterior) was not acquired. The postoperative DBS-electrode position serves as a surrogate marker for the intraoperative position because a DBS electrode is placed on a predefined microelectrode track after testing. ${ }^{41}$ No deviations with respect to the implanted trajectory were seen on postoperative inspection. We thus assume that the intraoperative positioning of test electrodes was accurate. Furthermore, intraoperative microrecordings and test stimulations showed typical results, indicating that the targeted tissue was stimulated at typical and planned electrode positions.

Clinical Detection of Capsular Effects as Opposed to Electrophysiological Measurements. One could argue that it would be more accurate to detect capsular adverse events with surface or needle electromyography. However, at the design stage of this study, we did not apply to our ethics committee for use of this method. In the clinical context, we believe, however, that a detection of the medial border of the CST, as performed every day during STN surgery, is accurate enough to draw conclusions.

Simulation of the Electric Field. We are implicitly using 2 simplified versions of electric field simulations: Both simulations look at the electric fields as rather spherelike structures. In our first modeling approach (EPio), empirical data from the literature was used. We were able to extract an empirical distance/current amplitude $(\mathrm{mm} / \mathrm{mA})$ relation from their analysis and applied it to our intraoperative electrophysiological distance measurements. ${ }^{20}$ In the second modeling approach (EPpo), we used a voltage- and impedance-driven model that was developed on the electrode geometry also used here. ${ }^{19,21}$ These models assume that the current density field surrounding the active contact of a DBS electrode unfolds in tissues that have an equal distribution of impedances throughout their volume. We know that this is not true and that the electric field is not likely to be optimally represented with a spherelike simulation because it will look more deformed and deflected in reality. ${ }^{21}$ However, if one concludes that the current density field expands uniformly on all sides until it is deflected by a larger fiber tract, the assumption of a spherelike electric field is reasonable. ${ }^{19}$ Most interesting, both approaches (the currentdriven one and the voltage- and impedance-driven approach) come to almost the same results when looking at concordance to DTI FT.

\section{CONCLUSIONS}

DTI FT depicted the medial CST border in concordance with electrophysiology under 2 different conditions and modeling approaches (EPio and EPpo). Under both conditions, electrophysiologic measurements are clearly related to the DTI FT. It is not possible to directly draw conclusions on the application accuracy of DTI FT itself from our data. One of the reasons is the inherent limitation of the electrophysiologic methods to detect the medial CST border (thickness of fibers; do we really stimulate most medial fibers in the CST?). Our data show that electrophysiology and DTI FT are concordant with \pm 2.44 and $\pm 2.5 \mathrm{~mm}$ (95\% limits of agreement) around the mean difference of the 2 measurements, respectively. Given that related to the clinical approach, only measurements in a range of $1-5 \mathrm{~mm}$ were possible, the actual value of the levels of agreement have to be critically judged. Although one can assume that there is some concordance, are the clinical concordance levels acceptable? Assuming that electrophysiology serves as a criterion standard, true mean distance values of 1-5 $\mathrm{mm}$ are plausible. The placement error of a stereotactic frame can range up to $2 \mathrm{~mm}$ in the single directions $(\mathrm{x}, \mathrm{y}, \mathrm{z})$, and a mean vector error can be as high as $3.15 \mathrm{~mm} .^{29}$

Assuming that there is a certain clinical dependence between the limits of agreement and the accuracy of the DTI FT method, a maximum error of $\pm 2.5 \mathrm{~mm}$ would be acceptable, especially in light of a 5-mm error in previous surgical DTI studies. ${ }^{15,17,28}$ Multiple factors add up to a combined application accuracy, and the present study was not designed to look at the accuracy but at concordance of 2 methods to determine the unknown value that expresses the distance between an electrode and the CST. Our own clinical experience with DTI FT-assisted targeting is that such an error, in reality, is smaller than our $2.5 \mathrm{~mm}$; thus, in interpreting the data here, we are likely dealing with inherent limitations of the method applied, which we tried to discuss above. In any case, our results are within the framework of our own previously published and postulated simulation studies for DBS fiber tracts and electric fields. ${ }^{19}$

Scientific groups, including ours, successfully use the DTI FT technology to assist functional neurosurgical procedures..$^{2-4,7,8,36,42,43}$ With all caution, our results would allow the further use of DTI FT to assist DBS procedures and to explore the effects and adverse events of DBS and lesion surgery. ${ }^{1,6,19,35,36}$ The future will show whether the development leads to a broader application of these direct DTI FT planning strategies based on individual "functional" anatomy. Clinical studies that investigate this technology are underway and are the focus of our ongoing research (www.clinicaltrials.gov; Deep braIn Stimulation for Tremor TractographIC Versus Traditional, NCT02491554; One Pass thalamIc aNd subthalamIc stimulation, NCT02288468).

Disclosures: Volker A. Coenen-UNRELATED: Consultancy: Dr Coenen has been a consultant for Medtronic (neuromodulation); Grants/Grants Pending: German Research Foundation, ${ }^{*}$ BrainLinks-BrainTools (cluster of excellence), ${ }^{\star}$ Wilhelm-Tönnis Foundation; Payment for Lectures (including service on Speakers Bureaus): Dr Coenen has occasionally received travel fees and honoraria from Medtronic, Boston Scientific; OTHER RELATIONSHIPS: Dr Coenen has ongoing Investigator Initiated Trials with Boston Scientific and Medtronic; OTHER: V.A.C. acted as a clinical consultant in the evaluation of StealthViz DTI (Medtronic Navigation) in this defined project between the University of British Columbia and Medtronic Navigation. V.A.C. has 
received limited funding as Principal Investigator for Investigator Initiated Trials from Medtronic and Boston Scientific. V.A.C. was supported with a stipend from the Wilhelm Tönnis Foundation from the German Society of Neurological Surgeons. Christopher R. Honey_UNRELATED: Grants/Grants Pending: Medtronic*; Payment for Lectures (including service on Speakers Bureaus): Medtronic*; OTHER: C.R.H. acted as a clinical consultant in the evaluation of StealthViz DTI (Medtronic Navigation) in this defined project between the University of British Columbia and Medtronic Navigation. Burkhard Mädler-UNRELATED: Employment: I am currently employed by Philips Healthcare, who did not provide any financial support for this study. Data collection and analysis were already completed before I became an employee of Philips. Only the period of manuscript drafting coincides with my employment status. *Money paid to the institution.

\section{REFERENCES}

1. Coenen VA, Schlaepfer TE, Allert N, et al. Diffusion tensor imaging and neuromodulation: DTI as key technology for deep brain stimulation. Int Rev Neurobiol 2012;107:207-34 CrossRef Medline

2. Henderson JM. "Connectomic surgery": diffusion tensor imaging (DTI) tractography as a targeting modality for surgical modulation of neural networks. Front Integr Neurosci 2012;6:15 CrossRef Medline

3. Barkhoudarian G, Klochkov T, Sedrak M, et al. A role of diffusion tensor imaging in movement disorder surgery. Acta Neurochir (Wien) 2010;152:2089-95 CrossRef Medline

4. Coenen VA, Allert N, Mädler B. A role of diffusion tensor imaging fiber tracking in deep brain stimulation surgery: DBS of the dentato-rubro-thalamic tract (drt) for the treatment of therapy-refractory tremor. Acta Neurochir (Wien) 2011;153:1579-85; discussion 1585 CrossRef Medline

5. Coenen VA, Fromm C, Kronenburger M, et al. Electrophysiological proof of diffusion-weighted imaging-derived depiction of the deepseated pyramidal tract in human. Zentralbl Neurochi 2006;67: 117-22 CrossRef Medline

6. Coenen VA, Honey CR, Hurwitz T, et al. Medial forebrain bundle stimulation as a pathophysiological mechanism for hypomania in subthalamic nucleus deep brain stimulation for Parkinson's disease. Neurosurgery 2009;64:1106-14; discussion 1114-15 CrossRef Medline

7. Coenen VA, Kieselbach K, Mader I, et al. Diffusion tensor magnetic resonance imaging (DTI) tractography-guided deep brain stimulation in neuropathic pain. Acta Neurochir (Wien) 2015;157:739-41 CrossRef Medline

8. Coenen VA, Mädler B, Schiffbauer H, et al. Individual fiber anatomy of the subthalamic region revealed with diffusion tensor imaging: $a$ concept to identify the deep brain stimulation target for tremor suppression. Neurosurgery 2011;68:1069-75; discussion 1075-76 CrossRef Medline

9. Coenen VA, Schlaepfer TE, Maedler B, et al. Cross-species affective functions of the medial forebrain bundle-implications for the treatment of affective pain and depression in humans. Neurosci Biobehav Rev 2011;35:1971-81 CrossRef Medline

10. Schlaepfer TE, Bewernick BH, Kayser S, et al. Rapid effects of deep brain stimulation for treatment-resistant major depression. Biol Psychiatry 2013;73:1204-12 CrossRef Medline

11. Coenen VA, Krings T, Mayfrank L, et al. Three-dimensional visualization of the pyramidal tract in a neuronavigation system during brain tumor surgery: first experiences and technical note. Neurosurgery 2001;49:86-92; discussion 92-83 Medline

12. Coenen VA, Krings T, Weidemann J, et al. Sequential visualization of brain and fiber tract deformation during intracranial surgery with three-dimensional ultrasound: an approach to evaluate the effect of brain shift. Neurosurgery 2005;56:133-41; discussion 133-41 Medline

13. Wu JS, Zhou LF, Tang WJ, et al. Clinical evaluation and followup outcome of diffusion tensor imaging-based functional neuronavigation: a prospective, controlled study in patients with gliomas involving pyramidal tracts. Neurosurgery 2007;61:935-48; discussion 948-49 CrossRef Medline

14. Kinoshita M, Yamada K, Hashimoto N, et al. Fiber-tracking does not accurately estimate size of fiber bundle in pathological condition: initial neurosurgical experience using neuronavigation and subcortical white matter stimulation. Neuroimage 2005;25:424-29 CrossRef Medline

15. Hahn HK, Klein J, Nimsky C, et al. Uncertainty in diffusion tensor based fibre tracking. Acta Neurochir Suppl 2006;98:33-41 CrossRef Medline

16. Nimsky C, Ganslandt O, Buchfelder M, et al. Intraoperative visualization for resection of gliomas: the role of functional neuronavigation and intraoperative 1.5 T MRI. Neurol Res 2006;28:482-87 CrossRef Medline

17. Nimsky C, Ganslandt O, Fahlbusch R. Implementation of fiber tract navigation. Neurosurgery 2006;58:ONS-292-303; discussion ONS303-04 Medline

18. Nimsky C, Ganslandt O, Hastreiter P, et al. Preoperative and intraoperative diffusion tensor imaging-based fiber tracking in glioma surgery. Neurosurgery 2007;61:178-85; discussion 186 Medline

19. Mädler B, Coenen VA. Explaining clinical effects of deep brain stimulation through simplified target-specific modeling of the volume of activated tissue. AJNR Am J Neuroradiol 2012;33:1072-80 CrossRef Medline

20. Ranck JB Jr. Which elements are excited in electrical stimulation of mammalian central nervous system: a review. Brain Res 1975;98: 417-40 CrossRef Medline

21. Butson CR, Maks CB, McIntyre CC. Sources and effects of electrode impedance during deep brain stimulation. Clin Neurophysiol 2006; 117:447-54 CrossRef Medline

22. Burgel U, Madler B, Honey CR, et al. Fiber tracking with distinct software tools results in a clear diversity in anatomical fiber tract portrayal. Cent Eur Neurosurg 2009;70:27-35 CrossRef Medline

23. Yousry TA, Schmid UD, Alkadhi H, et al. Localization of the motor hand area to a knob on the precentral gyrus: a new landmark. Brain 1997;120(pt 1):141-57 CrossRef Medline

24. Bland JM, Altman DG. Statistical methods for assessing agreement between two methods of clinical measurement. Lancet 1986;1: 307-10 Medline

25. Bland JM, Altman DG. Agreement between methods of measurement with multiple observations per individual. J Biopharm Stat 2007;17:571-82 CrossRef Medline

26. Nimsky C, Ganslandt O, Merhof D, et al. Intraoperative visualization of the pyramidal tract by diffusion-tensor-imaging-based fiber tracking. Neuroimage 2006;30:1219-29 CrossRef Medline

27. Nimsky C, Ganslandt O, von Keller B, et al. Intraoperative high-field MRI: anatomical and functional imaging. Acta Neurochir Suppl 2006;98:87-95 CrossRef Medline

28. Nimsky C, Ganslandt O, Fahlbusch R. Implementation of fiber tract navigation. Neurosurgery 2007;61:306-17; discussion 317-18 CrossRef Medline

29. Holloway KL, Gaede SE, Starr PA, et al. Frameless stereotaxy using bone fiducial markers for deep brain stimulation. J Neurosurg 2005; 103:404-13 CrossRef Medline

30. Bjartmarz H, Rehncrona S. Comparison of accuracy and precision between frame-based and frameless stereotactic navigation for deep brain stimulation electrode implantation. Stereotact Funct Neurosurg 2007;85:235-42 CrossRef Medline

31. Said N, Elias WJ, Raghavan P, et al. Correlation of diffusion tensor tractography and intraoperative macrostimulation during deep brain stimulation for Parkinson disease. J Neurosurg 2014;121: 929-35 CrossRef Medline

32. Coenen VA, McIntyre CC. Letter to the Editor: Correlation of diffusion tensor imaging and intraoperative macrostimulation. J Neurosurg 2015;123:291-92 CrossRef Medline

33. Chaturvedi A, Butson CR, Lempka SF, et al. Patient-specific models of deep brain stimulation: influence of field model complexity on neural activation predictions. Brain Stimul 2010;3:65-67 CrossRef Medline

34. Duerden EG, Finnis KW, Peters TM, et al. Three-dimensional somatotopic organization and probabilistic mapping of motor responses from the human internal capsule. J Neurosurg 2011;114: 1706-14 CrossRef Medline 
35. Sajonz B, Mädler B, Herberhold S, et al. A case of tremor reduction and almost complete ageusia under bilateral thalamic (VIM) deep brain stimulation in essential tremor-a therapeutic dilemma. Acta Neurochir (Wien) 2011;153:2361-63 CrossRef Medline

36. Sweet JA, Walter BL, Gunalan K, et al. Fiber tractography of the axonal pathways linking the basal ganglia and cerebellum in Parkinson disease: implications for targeting in deep brain stimulation. J Neurosurg 2014;120:988-96 CrossRef Medline

37. Kreher BW, Mader I, Kiselev VG. Gibbs tracking: a novel approach for the reconstruction of neuronal pathways. Magn Reson Med 2008; 60:953-63 CrossRef Medline

38. Mori S, Kaufmann WE, Davatzikos C, et al. Imaging cortical association tracts in the human brain using diffusion-tensor-based axonal tracking. Magn Reson Med 2002;47:215-23 CrossRef Medline

39. Wakana S, Jiang H, Nagae-Poetscher LM, et al. Fiber tract-based atlas of human white matter anatomy. Radiology 2004;230:77-87 CrossRef Medline

40. Pinsker MO, Herzog J, Falk D, et al. Accuracy and distortion of deep brain stimulation electrodes on postoperative MRI and CT. Zentralbl Neurochir 2008;69:144-47 CrossRef Medline

41. Shin M, Lefaucheur JP, Penholate MF, et al. Subthalamic nucleus stim- ulation in Parkinson's disease: postoperative CT-MRI fusion images confirm accuracy of electrode placement using intraoperative multiunit recording. Neurophysiol Clin 2007;37:457-66 Medline

42. Coenen VA, Allert N, Paus S, et al. Modulation of the cerebellothalamo-cortical network in thalamic deep brain stimulation for tremor: a diffusion tensor imaging study. Neurosurgery 2014;75: 657-69; discussion 669-70 CrossRef Medline

43. Sajonz BE, Mädler B, Herberhold S, et al. Stimulation induced hypogeusia in thalamic deep brain stimulation for tremor: an underestimated yet common side effect. J Neurol Neurosurg Psychiatry 2015 Apr 21. [Epub ahead of print] CrossRef Medline

44. Coenen VA, Abdel-Rahman A, McMaster J, et al. Minimizing brain shift during functional neurosurgical procedures: a simple burr hole technique that can decrease CSF loss and intracranial air. Cent Eur Neurosurg 2011;72:181-85 CrossRef Medline

45. Elias WJ, Fu KM, Frysinger RC. Cortical and subcortical brain shift during stereotactic procedures. J Neurosurg 2007;107:983-88 CrossRef Medline

46. Schaltenbrand W, Wahren. Atlas of Stereotaxy of the Human Brain, Stuttgart: Georg Thieme-Verlag; 1977 